PROCEEDINGS OF THE

AMERICAN MATHEMATICAL SOCIETY

Volume 128, Number 9, Pages 2801-2807

S 0002-9939(00)05343-0

Article electronically published on March 1, 2000

\title{
MICROBUNDLES, MANIFOLDS AND METRISABILITY
}

\author{
DAVID GAULD AND SINA GREENWOOD \\ (Communicated by Alan Dow)
}

\begin{abstract}
The notion of a microbundle was introduced in the 1960s but the theory came to an abrupt halt when it was shown that for a metrisable manifold, microbundles are equivalent to fibre bundles. In this paper we consider microbundles over non-metrisable manifolds. In some cases microbundles are equivalent to fibre bundles but in others they are not. In particular, we show that a manifold is metrisable if and only if its tangent microbundle is equivalent to a fibre bundle. We also illustrate that for some non-metrisable manifolds every trivial microbundle contains a trivial fibre bundle whereas other manifolds may support a trivial microbundle not containing a trivial fibre bundle.
\end{abstract}

\section{Definitions And notation}

Throughout this paper, by a manifold we mean a connected Hausdorff space in which each point has a neighbourhood homeomorphic to euclidean space. It is wellknown (cf. [4, p. 637]) that a manifold is metrisable if and only if it satisfies any one (and hence all) of the following properties: paracompact; $\sigma$-compact; second countable; meta-Lindelöf.

In 1964 Milnor 3 introduced the notion of a microbundle as a means of transferring some of the procedures applicable to bundles over smooth manifolds to manifolds which are not necessarily smooth. The development came to an abrupt halt when Kister 22 showed that over metrisable manifolds every microbundle is equivalent to a fibre bundle. At the time the major effort in the study of manifolds was concentrated on compact manifolds and, as far as we know, the study of non-metrisable manifolds did not begin systematically until the late 1970s.

Definition ([3]). A microbundle, denoted $B \stackrel{i}{\longrightarrow} E \stackrel{j}{\longrightarrow} B$, consists of topological spaces $B$ and $E$, called the base space and the total space respectively, and continuous functions $i$ and $j$, called the injection and projection maps respectively, such that the following conditions hold:

- $j i=1_{B}$, the identity map on $B$; and

- there is an open cover $\mathcal{U}$ of $B$ so that for each $U \in \mathcal{U}$ there are a set $V \subset$ $j^{-1}(U)$, with $i(U) \subset V$, and a homeomorphism

$$
\varphi_{U}: V \longrightarrow U \times \mathbb{R}^{n}
$$

Received by the editors July 8, 1997 and, in revised form, October 16, 1998

2000 Mathematics Subject Classification. Primary 57N55, 54E35, 55R60, 57N05, 57N15.

Key words and phrases. Metrisability, microbundle, non-metrisable manifold, tangent microbundle.

The second author's research was supported in part by a Marsden Fund Award, UOA611. 
such that the following diagram commutes:

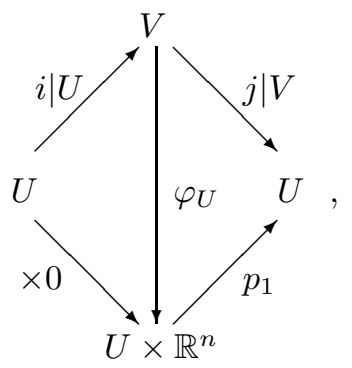

where $\times 0: U \longrightarrow U \times \mathbb{R}^{n}$ is defined by $(\times 0)(u)=(u, 0)$, and $p_{1}$ is projection on the first coordinate.

Definition. For any base space $B$, and any $n \geq 0, B \stackrel{\times 0}{\longrightarrow} B \times \mathbb{R}^{n} \stackrel{p_{1}}{\longrightarrow} B$ is called the standard trivial microbundle.

Definition. For any manifold $M$ the diagram

$$
M \stackrel{\Delta}{\longrightarrow} M \times M \stackrel{p_{1}}{\longrightarrow} M,
$$

where $\Delta$ is the diagonal map, constitutes a microbundle; it is called the tangent microbundle.

Definition. Two microbundles $B \stackrel{i_{1}}{\longrightarrow} E_{1} \stackrel{j_{1}}{\longrightarrow} B$ and $B \stackrel{i_{2}}{\longrightarrow} E_{2} \stackrel{j_{2}}{\longrightarrow} B$ are equivalent if there exist neighbourhoods $W_{1}$ of $i_{1}(B)$ in $E_{1}$ and $W_{2}$ of $i_{2}(B)$ in $E_{2}$, and a homeomorphism $\Phi: W_{1} \longrightarrow W_{2}$ such that the following diagram commutes:

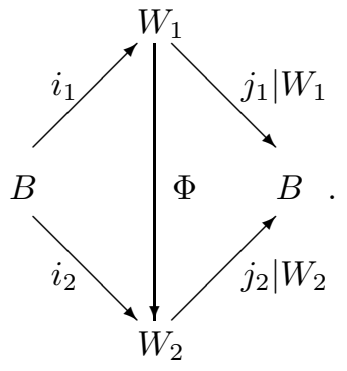

Definition. A microbundle which is equivalent to the standard trivial bundle is called a trivial microbundle.

We will denote the fibre bundle with base space $B$, total space $E$, 0 -section $e$ and bundle projection $p$ by $B \stackrel{e}{\longrightarrow} E \stackrel{p}{\longrightarrow} B$. In the following, fibre bundles will be assumed to have a 0 -section and fibre $\mathbb{R}^{n}$.

A fibre bundle satisfies all of the requirements of a microbundle. Kister's result 2 shows that if the base manifold is metrisable, then any microbundle admits a fibre bundle which is microbundle equivalent to the original microbundle. We address the question in the case where the base manifold is not metrisable.

Some of the results in this paper have been described in the authors' paper [1].

\section{TRIVIAL MiCROBUndLES MAY OR MAY NOT BE EQUIVALENT TO TRIVIAL BUNDLES}

Example 1. For a particular non-metrisable manifold it may be the case that every trivial microbundle over the manifold contains a trivial fibre bundle. 
Milnor [3] showed that if the base space $B$ of a trivial microbundle is paracompact, then some open subset of the total space is homeomorphic to all of $B \times \mathbb{R}^{n}$. In this case there is an equivalent fibre bundle. This is also the case for any trivial microbundle over the long ray $\mathbb{L}^{+}$since, by the pressing down lemma, any neighbourhood of $\mathbb{L}^{+} \times\{0\}$ in $\mathbb{L}^{+} \times \mathbb{R}$ must contain a long tube, i.e. a set $\left(\alpha, \omega_{1}\right) \times(-h, h)$ for some $\alpha \in \omega_{1}$ and some positive $h \in \mathbb{R}$.

Example 2. There is a manifold supporting a trivial microbundle which does not contain a trivial fibre bundle with the same injection and projection.

Consider the manifold $M$ (allied to the Prüfer manifold) found in [4, Example 3.6] except that we interchange the $x$ - and $y$-axes. Let

$$
A=\{(x, y): x, y \in \mathbb{R} \text { and } x \neq 0\} \text { and for each } y \in \mathbb{R} \text {, let } B_{y}=\{(0, y)\} \times \mathbb{R} .
$$

We will write $\langle y, z\rangle$ for a typical member $((0, y), z)$ of $B_{y}$. Set $M=A \cup\left(\bigcup_{y \in \mathbb{R}} B_{y}\right)$. $M$ is topologised by using the usual topology on $A$ and replacing each point, $(0, y)$, of the missing $y$-axis by the copy, $B_{y}$, of the real line. More precisely, suppose that $\langle y, z\rangle \in B_{y}$. Declare

$$
\left\{\left\{\langle y, \zeta\rangle \in B_{y}:|z-\zeta|<1 / n\right\} \cup T(y, z, n): n \in \mathbb{N}\right\}
$$

to be a neighbourhood basis at $\langle y, z\rangle$, where

$$
T(y, z, n)=\left\{(\xi, \eta) \in \mathbb{R}^{2}: 0<|\xi|<\frac{1}{n} \text { and } z-\frac{1}{n}<\frac{\eta-y}{|\xi|}<z+\frac{1}{n}\right\} .
$$

The triangles $T(y, z, n)$ are bounded by segments of rays emanating from $(0, y)$ with slopes $\pm\left(z-\frac{1}{n}\right)$ and $\pm\left(z+\frac{1}{n}\right)$, where the \pm sign is determined by which side of the $y$-axis we are looking at. Because $M$ contains an uncountable discrete closed subset it cannot be Lindelöf, hence cannot be metrisable.

Define an open subset $E$ of $M \times(-1,1)$ by

$$
E=[A \times(-1,1)] \cup\left\{(\langle y, z\rangle, t) \in B_{y} \times(-1,1): y \in \mathbb{R} \text { and }|t|<\min \left\{1, \frac{1}{|z|}\right\}\right\} .
$$

Then $M \stackrel{\times 0}{\longrightarrow} E \stackrel{p_{1}}{\longrightarrow} M$ is a trivial microbundle which does not contain a trivial fibre bundle.

Suppose to the contrary that this microbundle did contain a trivial fibre bundle. Then there would be a continuous function $f: M \longrightarrow(0,1)$ so that

$$
\forall p \in M \text { and } \forall t \in(-1,1),|t|<f(p) \Rightarrow(p, t) \in E .
$$

Such a function may be obtained from a trivial fibre bundle as follows: if $M \stackrel{\times 0}{\longrightarrow}$ $D \stackrel{p_{1}}{\longrightarrow} M$ is a trivial fibre bundle, then there is a fibre-preserving homeomorphism $h: M \times \mathbb{R} \longrightarrow D$, so we may define $f$ by letting $f(p)$ be the second coordinate of $h(p, 1)$.

For each $m, n \in \mathbb{N}$ set $S(m, n)=\left\{y \in \mathbb{R}: f(T(y, 0, n)) \subset\left(\frac{1}{m}, 1\right)\right\}$. Then $\bigcup_{m, n \in \mathbb{N}} S(m, n)=\mathbb{R}$ because for each $y \in \mathbb{R}$ continuity of $f$ implies that there are integers $m, n \in \mathbb{N}$ such that $f(T(y, 0, n)) \subset\left(\frac{1}{m}, 1\right)$. Since the union is countable, $S(m, n)$ is uncountable for some $m, n \in \mathbb{N}$; suppose we have chosen such $m, n$. As an uncountable subset of $\mathbb{R}$, the set $S(m, n)$ contains a point $a$ which is a limit point of $S(m, n) \cap(a, \infty)$. Thus $f(T(a, 0, n)) \subset\left(\frac{1}{m}, 1\right)$. By the definition of $E$ we have that $f(\langle a, m\rangle)<\frac{1}{m}$, so there is $l \in \mathbb{N}$ so that $f(T(a, m, l)) \subset\left(0, \frac{1}{m}\right)$; we may assume that $l \geq n$. Pick $(x, y) \in T(a, m, l)$. Then $y>a$ and as $a$ is a limit point of $S(m, n) \cap(a, \infty)$, there is $b \in S(m, n)$ with $a<b<y$; thus $f(T(b, 0, n)) \subset\left(\frac{1}{m}, 1\right)$. 
Because $a<b<y$ and $l \geq n$, it follows that $T(b, 0, n) \cap T(a, m, l) \neq \varnothing$, say $(u, v) \in T(b, 0, n) \cap T(a, m, l)$. On the one hand $(u, v) \in T(b, 0, n)$ so $f(u, v)>\frac{1}{m}$. On the other hand $(u, v) \in T(a, m, l)$ so $f(u, v)<\frac{1}{m}$, a contradiction.

Thus, such a continuous function as $f$ cannot exist, so the trivial microbundle $M \longrightarrow E \longrightarrow M$ does not contain a trivial fibre bundle.

Example 3. Two fibre bundles may be equivalent as microbundles while not being equivalent as fibre bundles. Indeed, the following fibre bundle is microbundle equivalent to the standard trivial microbundle but is not fibre bundle equivalent to the trivial fibre bundle.

Let $\mathbb{L}^{+} \stackrel{e}{\longrightarrow} \mathbb{L}^{+} \times \mathbb{L} \stackrel{p}{\longrightarrow} \mathbb{L}^{+}$, where $\mathbb{L}^{+}$is the long ray and $\mathbb{L}$ is the long line, be the fibre bundle in which $p$ maps $(x, y)$ to $x$ if $x \geq|y|$ and to $|y|$ otherwise. Note that $\left\{(0, \alpha): \alpha \in \omega_{1}\right\}$ covers $\mathbb{L}^{+}$and that each $p^{-1}(0, \alpha)$ is homeomorphic to $(0, \alpha) \times \mathbb{R}$. This bundle is microbundle equivalent to the standard trivial microbundle, for we may let

$$
W_{1}=W_{2}=\left\{(x, y) \in \mathbb{L}^{+} \times \mathbb{L}:|y|<x \leq 1\right\} \cup\left\{(x, y) \in \mathbb{L}^{+} \times \mathbb{L}:|y|<1 \leq x\right\},
$$

and $\Phi: W_{1} \longrightarrow W_{2}$ be the identity. However it is not equivalent to the trivial fibre bundle as $\mathbb{L}^{+} \times \mathbb{L}$ is not homeomorphic to $\mathbb{L}^{+} \times \mathbb{R}$.

\section{Microbundles AND METRISABility}

Recall that a topological space is defined to be meta-Lindelöf if and only if every open cover has a point-countable open refinement. Also (cf. [4, Theorem 2.5]) for a manifold the following conditions are equivalent: metrisable, paracompact, meta-Lindelöf, (hereditarily) Lindelöf, second countable and $\sigma$-compact.

Lemma 1. Let $M$ be a manifold and suppose that $M=\bigcup_{n \in \mathbb{N}} U_{n}$, where for each $n \in \mathbb{N}$ we have that $U_{n}$ is open and metrisable. Then $M$ is metrisable.

Proof. Let $\mathcal{V}$ be an open cover of $M$. For each $n \in \mathbb{N}$ let $\mathcal{U}_{n}=\left\{V \cap U_{n}: V \in \mathcal{V}\right\}$. Then $\mathcal{U}_{n}$ is an open cover of $U_{n}$. As $U_{n}$ is metrisable it is also metacompact, hence $\mathcal{U}_{n}$ has a point-finite open refinement, say $\mathcal{W}_{n}$. Let $\mathcal{W}=\bigcup_{n \in \mathbb{N}} \mathcal{W}_{n}$. Then $\mathcal{W}$ is a point-countable open refinement of $\mathcal{V}$. Thus $M$ is meta-Lindelöf and hence metrisable.

Theorem 1. Let $M^{m}$ be a manifold. Then the following conditions are equivalent:

(i) $M$ is metrisable;

(ii) there is an open cover $\mathcal{U}$ of $M$ such that for each $x \in M$ the set $\operatorname{st}(x, \mathcal{U})$ is homeomorphic to an open subset of $\mathbb{R}^{m}$;

(iii) there is an open cover $\mathcal{U}$ of $M$ such that for each $x \in M$ the set $\operatorname{st}(x, \mathcal{U})$ is metrisable;

(iv) there is an open cover $\mathcal{U}$ of $M$ such that for each $x \in M$ the set $\operatorname{st}(x, \mathcal{U})$ is Lindelöf;

(v) there is a cover $\mathcal{U}$ of $M$ such that for each $x \in M$ the set st $(x, \mathcal{U})$ is open and Lindelöf;

(vi) there is a cover $\mathcal{U}$ of $M$ such that for each $x \in M$ the set $\operatorname{st}(x, \mathcal{U})$ is open and metrisable.

Proof. (i) $\Longrightarrow$ (ii): Suppose that $M$ has a metric. As a metrisable manifold, $M$ is $\sigma$-compact, so may be expressed as a union of countably many compact subsets, 
say $M=\bigcup_{n \in \mathbb{N}} C_{n}$. We may assume that $C_{n} \subset \stackrel{\circ}{C}_{n+1}$ for each $n \in \mathbb{N}$. When $n \leq 0$, by $C_{n}$ we will mean the empty set.

By compactness we may cover $C_{n}-\stackrel{\circ}{C}_{n-1}$ by a finite family $\mathcal{V}_{n}$ of sets each of which is homeomorphic to an open subset of $\mathbb{R}^{m}$ and each of which is a subset of $\stackrel{\circ}{C}_{n+1}-C_{n-2}$. Let $\varepsilon_{n}>0$ be a Lebesgue number for $\mathcal{V}_{n}$, i.e. $\varepsilon_{n}$ is such that every ball of radius $\varepsilon_{n}$ and centre in $C_{n}-\stackrel{\circ}{C}_{n-1}$ lies in some member of $\mathcal{V}_{n}$. We let $\varepsilon_{0}=1$.

For each $n \in \mathbb{N}$, let $\mathcal{U}_{n}$ be a cover of $C_{n}-\stackrel{\circ}{C}_{n-1}$ by balls whose centres lie in $C_{n}-\stackrel{\circ}{C}_{n-1}$ and whose diameters are $\min \left\{\varepsilon_{n-1}, \varepsilon_{n}, \varepsilon_{n+1}\right\}$. Let $\mathcal{U}=\bigcup_{n \in \mathbb{N}} \mathcal{U}_{n}$. If $x \in M$, say $x \in C_{n}-\stackrel{\circ}{C}_{n-1}$, then $\operatorname{st}(x, \mathcal{U})$ is a union of balls from $\mathcal{U}_{n-1} \cup \mathcal{U}_{n} \cup \mathcal{U}_{n+1}$, hence of diameters at most $\varepsilon_{n}$. Thus $\operatorname{st}(x, \mathcal{U}) \subset B\left(x ; \varepsilon_{n}\right)$, so $\operatorname{st}(x, \mathcal{U})$ lies in some member of $\mathcal{V}_{n}$ so is homeomorphic to an open subset of $\mathbb{R}^{m}$.

(ii) $\Longrightarrow$ (iii): Obvious.

(iii) $\Longrightarrow\left(\right.$ iv): Let $\mathcal{U}^{\prime}$ be a cover as in (iii) and let

$$
\mathcal{U}=\left\{U \subset M: U \text { is a component of some member of } \mathcal{U}^{\prime}\right\} .
$$

Then each $U \in \mathcal{U}$ is connected and open; thus for each $x \in M, \operatorname{st}(x, \mathcal{U})$ is connected and open, and hence a manifold which is a subset of the metrisable manifold, $s t\left(x, \mathcal{U}^{\prime}\right)$. Thus $s t(x, \mathcal{U})$ is Lindelöf.

(iv) $\Longrightarrow(\mathrm{v})$ : Obvious.

$(\mathrm{v}) \Longrightarrow(\mathrm{vi}):$ Take $\mathcal{U}$ as in $(\mathrm{v})$. Then $s t(x, \mathcal{U})$ is $\mathrm{T}_{3}$ and Lindelöf, hence metrisable.

(vi) $\Longrightarrow(\mathrm{i})$ : Suppose that $\mathcal{U}$ is a cover of $M$ such that $\operatorname{st}(x, \mathcal{U})$ is open in $M$ and metrisable for each $x \in M$. First, we will show that the manifold contains a sequence $\left\langle V_{\alpha}: \alpha \in \omega_{1}\right\rangle$ of subsets of $M$ satisfying

- $V_{\alpha}$ is Lindelöf, connected and open for each $\alpha$,

- $\overline{V_{\beta}} \subset V_{\alpha}$ whenever $\beta<\alpha$, and

- $V_{\lambda}=\bigcup_{\alpha<\lambda} V_{\alpha}$ for each limit $\lambda \in \omega_{1}$

(i.e. $M$ is of Type I) as follows.

1. Let $V_{0}$ be any open subset of $M$ homeomorphic to $\mathbb{R}^{m}$.

2. If $V_{\alpha}$ is defined as above for any ordinal $\alpha$, then $V_{\alpha}$ is separable: let $D_{\alpha}$ be a countable dense subset. Note that $\bar{V}_{\alpha} \subset \bigcup_{d \in D_{\alpha}} s t(d, \mathcal{U})$, for given $x \in \bar{V}_{\alpha}$, choose $d \in D_{\alpha} \cap \operatorname{st}(x, \mathcal{U})$; then $x \in \operatorname{st}(d, \mathcal{U})$. Thus, because $V_{\alpha}$, hence $\bar{V}_{\alpha}$, is a connected set, it is contained in a single component of $\bigcup_{d \in D_{\alpha}} s t(d, \mathcal{U})$. Let $V_{\alpha+1}$ be the component of $\bigcup_{d \in D_{\alpha}} s t(d, \mathcal{U})$ containing $\bar{V}_{\alpha}$. By Lemma 1, the set $V_{\alpha+1}$ is Lindelöf. Clearly the other conditions required of $V_{\alpha+1}$ are also satisfied.

3. If $\lambda$ is any limit ordinal and $V_{\alpha}$ is defined for each $\alpha<\lambda$, then $V_{\lambda}=\bigcup_{\alpha<\lambda} V_{\alpha}$ is Lindelöf, connected and open, being an increasing countable union of such sets, and the other two conditions are clearly satisfied.

Note that $M=\bigcup_{\alpha \in \omega_{1}} V_{\alpha}$. Indeed, as a union of open sets, $\bigcup_{\alpha \in \omega_{1}} V_{\alpha}$ is open. On the other hand, $\bigcup_{\alpha \in \omega_{1}} V_{\alpha}$ is closed, for suppose $x \in \overline{\bigcup_{\alpha \in \omega_{1}} V_{\alpha}}$ and let $\left\{U_{n}: n \in \mathbb{N}\right\}$ be a countable neighbourhood base at $x$. For each $n \in \mathbb{N}$ choose $\beta_{n} \in \omega_{1}$ such that $U_{n} \cap V_{\beta_{n}} \neq \varnothing$. Letting $\beta=\sup \left\{\beta_{n}: n \in \mathbb{N}\right\}$, we have $U_{n} \cap V_{\beta} \neq \varnothing$ for each $n \in \mathbb{N}$ so that $x \in \bar{V}_{\beta}$ and hence $x \in V_{\beta+1}$, so $x \in \bigcup_{\alpha \in \omega_{1}} V_{\alpha}$. As a non-empty open and closed subset of the connected space $M, \bigcup_{\alpha \in \omega_{1}} V_{\alpha}$ is all of $M$. 
Let $\Lambda$ be the set of limit ordinals of $\omega_{1}$. If for some $\lambda \in \Lambda$ we have $\bar{V}_{\lambda}-V_{\lambda}=\varnothing$, then $V_{\lambda}$ is open and closed, so is all of $M$ (by connectedness) and hence $M$ is Lindelöf and hence metrisable.

Suppose instead that for each $\lambda \in \Lambda$ we have $\bar{V}_{\lambda}-V_{\lambda} \neq \varnothing$. Then $M$ cannot be metrisable but we will obtain a contradiction.

Choose $x_{\lambda} \in \bar{V}_{\lambda}-V_{\lambda}$. Define $f: \Lambda \longrightarrow \omega_{1}$ by

$$
f(\lambda)=\min \left\{\alpha \in \omega_{1}: \operatorname{st}\left(x_{\lambda}, \mathcal{U}\right) \cap V_{\alpha} \neq \varnothing\right\} .
$$

As $\operatorname{st}\left(x_{\lambda}, \mathcal{U}\right)$ is a neighbourhood of $x_{\lambda} \in \bar{V}_{\lambda}$ it follows that $f(\lambda)<\lambda$. Thus by the pressing down the lemma we may choose $\alpha \in \omega_{1}$ such that $A=f^{-1}(\alpha)$ is stationary.

Recall the dense subset $D_{\alpha}$ of $V_{\alpha}$. For each $\lambda \in A$ choose $d_{\lambda} \in \operatorname{st}\left(x_{\lambda}, \mathcal{U}\right) \cap D_{\alpha}$ and let $\operatorname{cst}_{\lambda}\left(d_{\lambda}, \mathcal{U}\right)$ denote the component of $s t\left(d_{\lambda}, \mathcal{U}\right)$ containing $x_{\lambda}$ : note that $\operatorname{cst}_{\lambda}\left(d_{\lambda}, \mathcal{U}\right)$ is open and connected. Define $g: A \longrightarrow \omega_{1}$ by

$$
g(\lambda)=\min \left\{\beta \in \omega_{1}: \operatorname{cst}_{\lambda}\left(d_{\lambda}, \mathcal{U}\right) \cap V_{\beta} \neq \varnothing\right\} .
$$

Again, we may apply the pressing down lemma to find $\beta \in \omega_{1}$ so that $B=g^{-1}(\beta)$ is stationary.

Because $\operatorname{cst}_{\lambda}\left(d_{\lambda}, \mathcal{U}\right) \cap D_{\beta} \neq \varnothing$ for each $\lambda \in B$, and $D_{\alpha}$ and $D_{\beta}$ are countable whereas $B$ is uncountable, we may choose $d \in D_{\alpha}$ and $d^{\prime} \in D_{\beta}$ such that

$$
C=\left\{\lambda \in B: d_{\lambda}=d \text { and } d^{\prime} \in \operatorname{cst}_{\lambda}\left(d_{\lambda}, \mathcal{U}\right)\right\}
$$

is uncountable. Thus for each $\lambda \in C$, we have that $\operatorname{cst}_{\lambda}(d, \mathcal{U})$ is a component of $s t(d, \mathcal{U})$. Also since $d^{\prime} \in \operatorname{cst}_{\lambda}(d, \mathcal{U})$, it follows that $N=\bigcup_{\lambda \in C} c s t_{\lambda}(d, \mathcal{U})$ is connected (in fact $N=\operatorname{cst}_{\lambda}(d, \mathcal{U})$ for each $\lambda \in C$ ). Then $N$ is a metrisable manifold, hence Lindelöf.

Let $X=\left\{x_{\lambda} \in M: \lambda \in C\right\}$. On the one hand, because $X \subset N$ and $N$ is hereditarily Lindelöf, $X$ is Lindelöf. On the other hand, $\left\{V_{\lambda}: \lambda \in \Lambda\right\}$ forms an open cover of $X$ with no countable subcover, and hence $X$ cannot be Lindelöf, a contradiction.

Theorem 2. The tangent microbundle over a manifold $M$ is microbundle equivalent to a fibre bundle if and only if $M$ is metrisable.

Proof. $\Longrightarrow$ : Suppose that the tangent microbundle, $M \stackrel{\Delta}{\longrightarrow} M \times M \stackrel{p_{1}}{\longrightarrow} M$, is equivalent to a fibre bundle $M \stackrel{e}{\longrightarrow} E \stackrel{p}{\longrightarrow} M$. Then there is an open neighbourhood $W$ of $\Delta(M)$ in $M \times M$, which is fibre-preserving homeomorphic to an open neighbourhood $W^{\prime}$ of $e(M)$ in $E$.

For each $x \in M$ let $F_{x}=\{y \in M:(x, y) \in W\}$, an open subset of $M$. As part of the fibre $p_{1}^{-1}(x)$ the set $F_{x}$ is homeomorphic to an open subset of $\mathbb{R}^{m}$. Also for each $x \in M$ choose an open subset $U_{x}$ of $M$ such that $U_{x} \times U_{x} \subset W$. Set $\mathcal{U}=\left\{U_{x}: x \in M\right\}$. Then $\mathcal{U}$ satisfies condition (ii) of Theorem $\mathbb{1}$ as $\forall x \in M$, $\operatorname{st}(x, \mathcal{U}) \subset F_{x}$.

$\Longleftarrow$ : If $M$ is metrisable, then every microbundle over $M$ admits a fibre bundle by [2, Corollary 1].

Remark. We may use the ideas of the proof of Theorem 2 to obtain an alternative proof of the (i) $\Longrightarrow$ (ii) part of the proof of Theorem 1 If $M$ is metrisable, then by [2, Corollary 1] there is a fibre bundle which may be embedded fibrewise as a neighbourhood of the diagonal in $M \times M$. Then the open cover $\mathcal{U}$ is obtained from the fibres of this fibre bundle. 


\section{ACKNOWLEDGEMENT}

We thank Abdul Mohamad for suggesting the equivalent conditions (v) and (vi) in Theorem 1 .

\section{REFERENCES}

[1] Sina Greenwood and David Gauld Microbundles revisited, Proceedings of Prague TOPOSYM 1996, Topology Atlas, (1997), 114-119. CMP 98:13

[2] James M. Kister Microbundles are Fibre Bundles. Ann. Math., 80(1964), 190-199. [MR 31:5216

[3] John Milnor Microbundles Part I. Topology, 3, suppl.1(1964), 53-80. MR 28:4553b

[4] Peter Nyikos The Theory of Nonmetrizable Manifolds, in Handbook of Set-theoretic Topology, K. Kunen and J.Vaughan, Eds, (1984), 633-684. MR 86f:54054

Department of Mathematics, University of Auckland, Private Bag 92019, Auckland, New Zealand

E-mail address: gauld@math.auckland.ac.nz

E-mail address: sina@math.auckland.ac.nz 